\title{
Multiple Tags Positioning Algorithm using ESP8266 NODEMCU System
}

\author{
EL ABKARI Safae ${ }^{1}$ and EL MHAMDI Jamal ${ }^{2}$ \\ ${ }^{1}$ Research Scholar and ${ }^{2}$ Professor \\ Department of Electrical Engineering \\ Ecole Normale Superieure of Technical Education \\ Mohamed V University \\ Rabat, Morocco
}

\begin{abstract}
The emergence of the Internet of things (IoT) shaped the future of many domains where connectivity became a hot topic in electronic engineering. Recently, IoT was being a part of Indoor positing systems where devices need to communicate continuously at a defined time slot leading to various problematics for the conventional positioning algorithm. We propose an ESP8266 NodeMCU tracking system of multiple tags using a Wireless communication. The proposed implemented algorithm consists of two-phase in the first stage, a master anchor sends a signal to tags in order to establish the communication. In the second one, the master anchor initiates distance ranging and positioning. This two-phase based system aims to minimize reading time and increase the accuracy of positioning by reducing distance ranging errors, which we validate in our proposed model and algorithm by an experiment in an indoor environment.
\end{abstract}

Key Words: Wireless, Distance ranging, Anchors, ESP8266 node.

\section{INTRODUCTION}

A demand for location technologies has been increasing with the advancement of technologies. In this manner, a positioning system refers to the determination of the location of an asset, a person or an object having a wireless link to a recognized node within a coordinate in two or three dimensions within a locating system [1]. Ideally, the positioning location is present everywhere, improve control of logistics and safety inside buildings, outside the street, underground mines, etc. Walls and ceilings block radio signals consequently signal level is extremely low and connectivity is poor, which lead to think of technologies other than space-based navigation regardless of their high accuracy. Various approaches in indoor have emerged as radio-frequency (RF) ID tags [2-4], Bluetooth [5], Magnetic field [6], Wi-Fi [7-8], etc. depending on the application.

Among indoor locating techniques, Wi-Fi exploits the received signal (RSSI) to determine positions [8]. Wi-Fi based locating system is free of additional infrastructure, specific hardware chips and is known to be accurate. In this type of system, a training phase is needed to construct a list of signature signal (database), identify tags and facilitate positioning. Based on the result of RSSI signal processing and calculation, we obtain the location of the user. However, RSSI signal fluctuation and variation remain the fundamental issue.

RSSI method calculates coordinates of tags localization based on geometric principles such as fingerprinting [9]. The coordinate description is related to the geometric shape of a space. Therefore, we ordinarily operate in an explicit space with coordinates as $(\mathrm{x}, \mathrm{y}, \mathrm{z})$ to represent a space model so as a position location information.

Our proposed ESP8266 Node MCU system is constituted of ESP8266 Node MCU modules and an implemented two consecutively phases algorithm. The first stage consists of member identification and registration where the master anchor sends a signal to each tag of the network to confirm tags communication ability with anchors. When the tags send a response signal to the master anchor, the communication status is then confirmed. The second stage starts after the tags are triggered by another generated signal from the master anchor. This phase can also be repetitive for a number of rounds defined by the user and the application. Compared to a mono-phase (conventional) system, this ESP8266 Node MCU positioning algorithm proves its effectiveness in a real model in our experiments. 


\section{ESP8266 NODEMCU TRACKING MODEL ALGORITHM}

\subsection{ESP8266 NODEMCU proposed system}

We conceive ESP8266 Node MCU wireless network using this module as anchors and active tags as shown in Figure 1. A network cell consists of a fixed number of anchors, portable tags, a master anchor, a sink node and a task manager. Anchors and tags are working under an IEEE802.11b standard. To successfully determine location of a tag by geometrical trilateration, at least three anchors are required (network cell). We use ESP8266 Node MCU as a sink node which manages the network among other anchors by ranging received data signals.

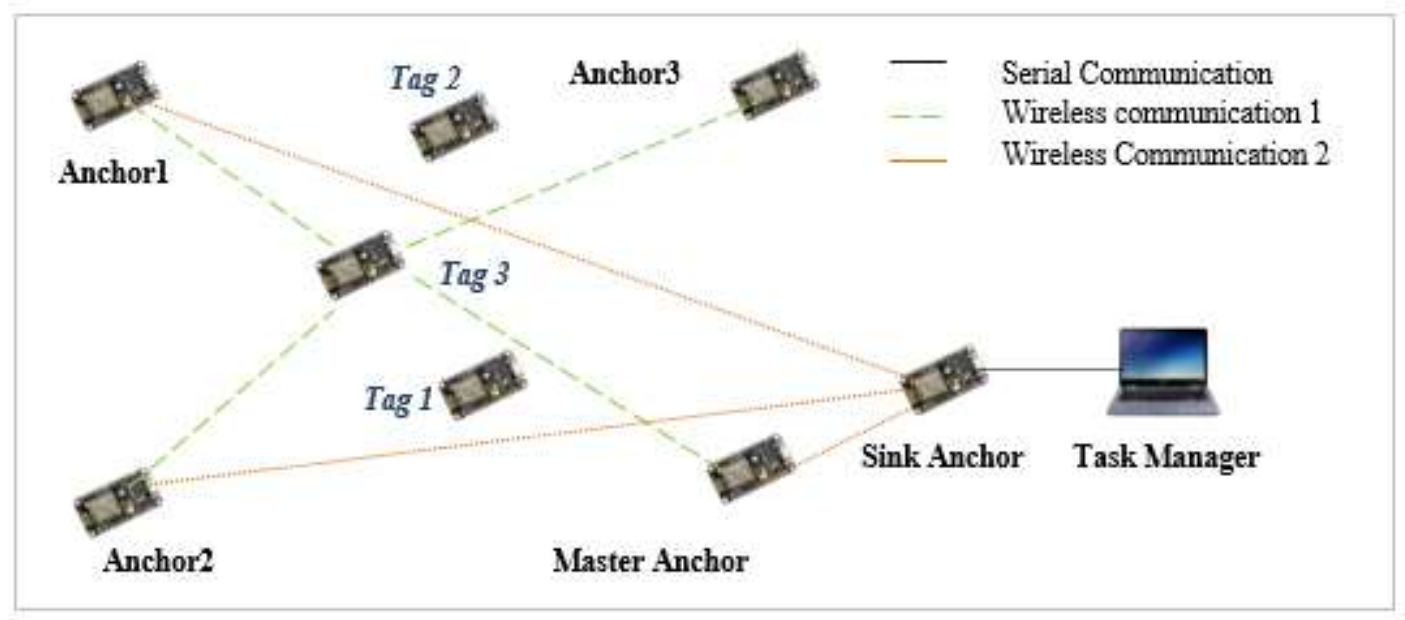

Figure 1: System configuration including network management

Tag identifier (ID) numbers are designated differently. A list of tags is installed in prior in the master anchor to compare it to the identified tags during the identification process.

\subsection{Proposed Algorithm}

Multiple ESP8266 Node MCU modules are assigned to their specific function in the network cell. ESP8266 NodeMCU master anchor mainly managed this algorithm composed of three stages, tags reading, distance ranging and network tracking.

Once the tags reading phase is executed, several positioning stages are operating depending on the number of existing tags and those identified during the previous stage. Tracking in the network comes as a final step in the repetitive routine loop presented and simplified in this block diagram (Figure 2).

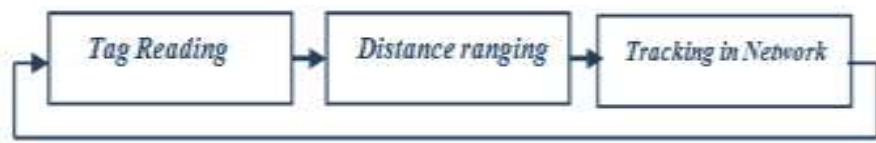

Figure 2: Block diagram of the tracking model process

The master anchor performs members registration, transmits signals to tags in to determine present tags in the reading range. The master anchor receives back a signal from this tag as a response signal to the check signal sent earlier if the tag can communicate with anchors.

In the positioning stage, the process is initiated by the response signal which is triggered by the signal of the master anchors. Tags are then registered to start network tracking by starting ranging with other anchors.

\subsection{Algorithm Details}

The first stage is tag reading where the master anchor sends a check signal to tags to determine their availability and capacity to communicate with anchors. Figure 3 block diagram shows the process of the master anchor at its reading stage. Tags receive a check signal from the master anchor to compare it to database stored data. Once a tag is activated, the master anchor receives back a check response signal to register the tag successfully in the loop as a member. 
The second stage is the positioning stage. We trigger identified and registered tags by the master anchor and start the positioning procedure. Figure 4 describes the aspects of the master anchor at ranging stage in a block diagram. The master anchor sends a signal as a call to each tag according to the list of members obtained in the reading phase. Frame structure of the check signal is identical for both call and check signal. The sufficient ranging time between the tag and anchors is guaranteed by the master anchor while other tags are in standby status. At the second stage, master anchor moves to a new member identification and registration (first stage) as soon as the distance ranging procedure is completed.

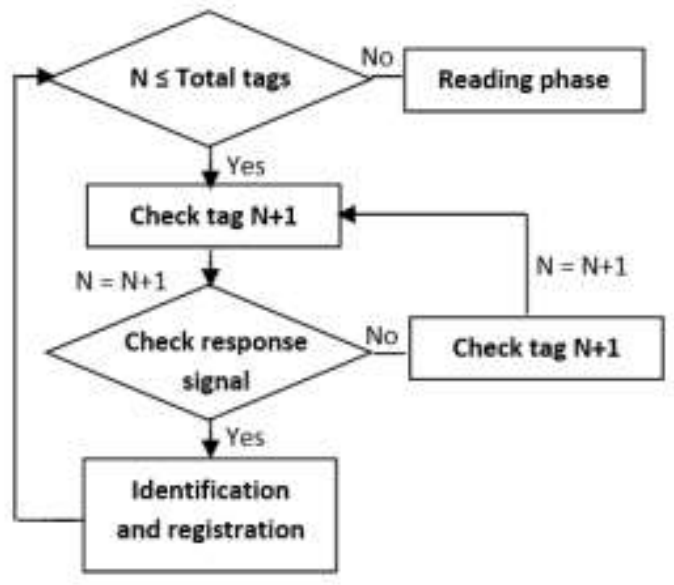

Figure 3: Master anchor block diagram at reading phase

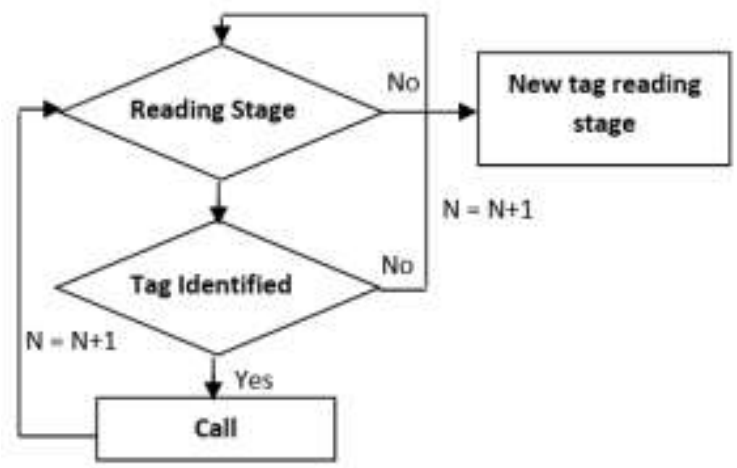

Figure 4: Master anchor diagram at ranging phase

\subsection{Phases of the proposed algorithm}

The ESP8266 NODEMCU based system implements the algorithm below with separate stages (tag reading phase and distance ranging) to obtain flexibility in time consumption and minimize time loss that occurs in systems within the second stage.

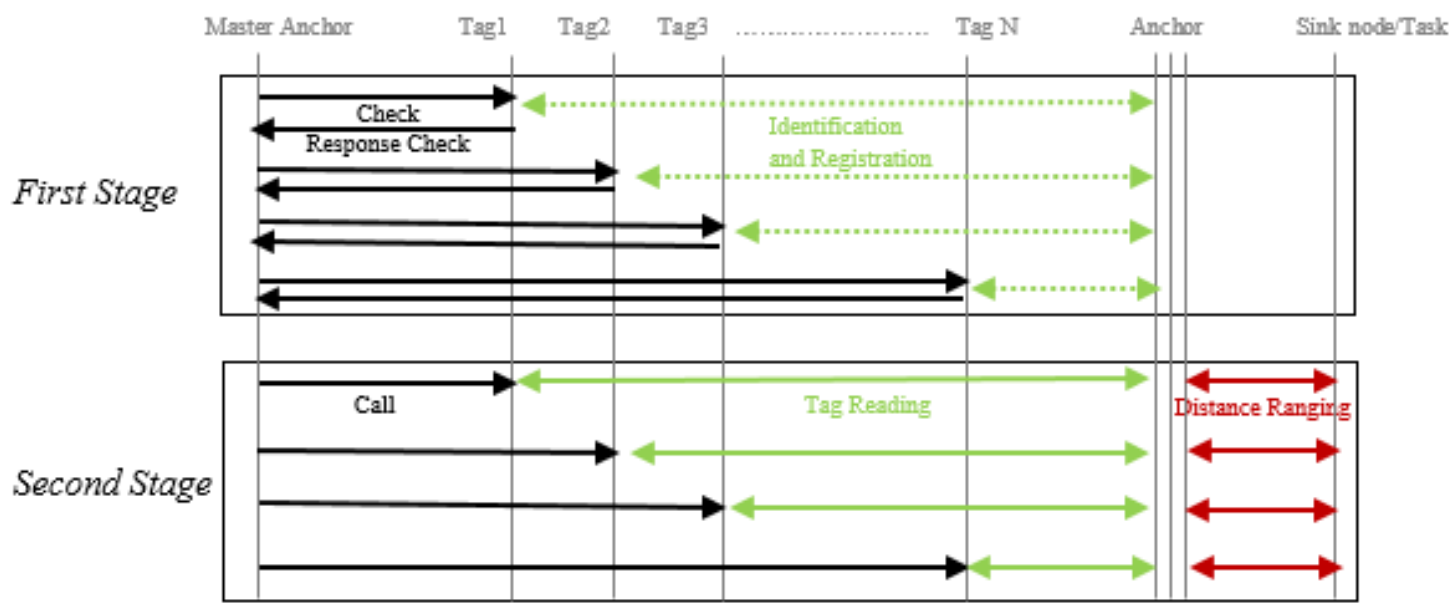

Figure 5: the implemented algorithm time flow 


\section{ESP8266 NODEMCU NETWORK}

\subsection{Algorithm process}

Initially, master anchor identifies and registers tags. Sink node receives distance values by reassembling data of anchors. Accordingly, the task manager receives the processed ranging data from the sink node to start positioning.

Positions within a network are determined as single coordinates in two-dimensional spaces with at least three distances from the anchors. Not only tag movement during distance ranging phase can create position imperfect but also the variation of RSSI values in consecutive ranging procedures cause additional positioning error. Therefore, we implement the two-phase algorithm to reduce errors and enhance our system performance. For our experimental model, we place the anchors and tag at the similar level in our experiment to maximize Line-Of-Sight anchors.

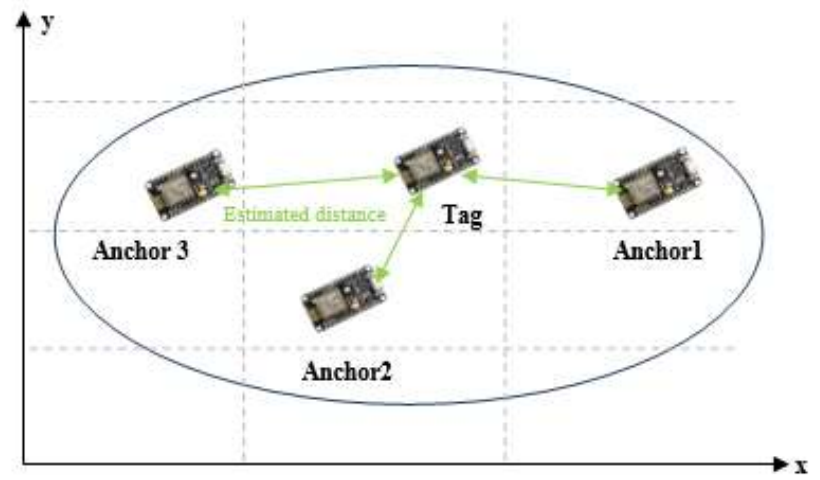

Figure 6: distance ranging of a tag in our network cell

We collect multiple RSSI values at a position to determine precise tag distance to anchors. We use then curve fitting technique (CFT) method because of its reliability in ranging [9]. CFT method is simple and applicable for active tag positioning. Thus, it provides better results compared to the Friis transmission and equation for free space propagation (FTE) estimation methods.

\subsection{Environment}

We implement the two-phase algorithm in the ESP8266 NodeMCU network cell constituted of anchors, master anchors, sink node, task manager, tag. We use a personal computer as a sink node/task manager, three ESP8266 NODEMCU wireless modules are configured as anchors and an additional one as a tag. We set our experiment environment in an indoor room (Figure 7).

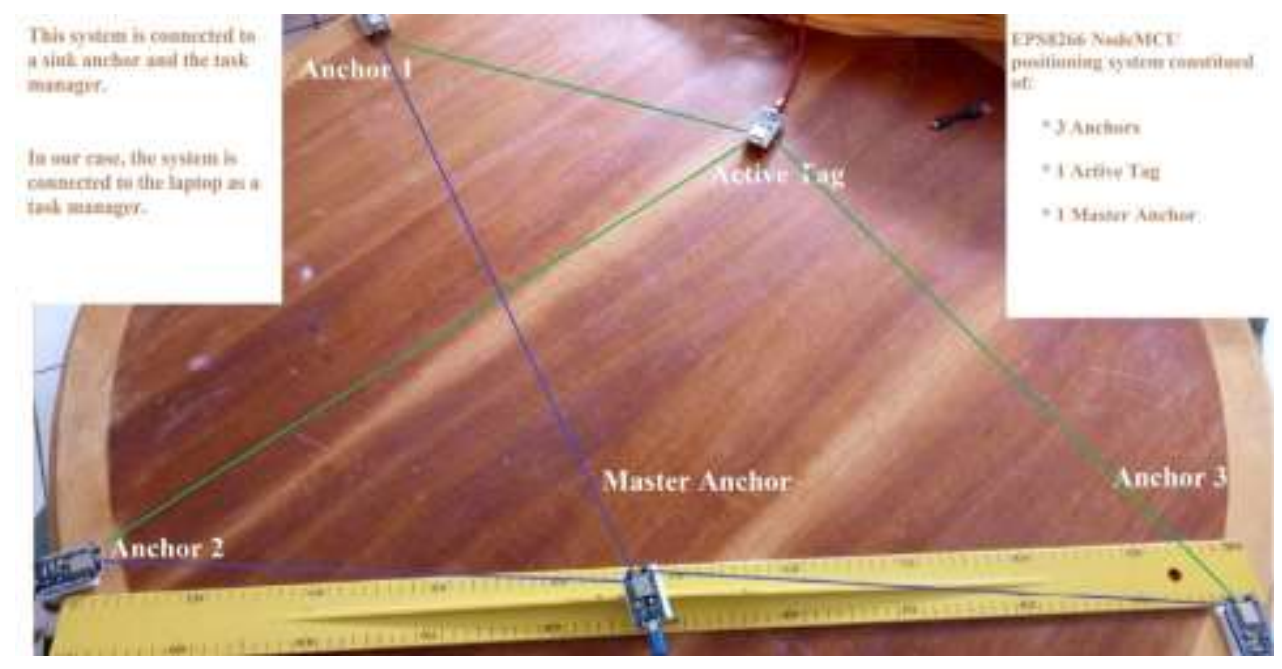

Figure 7: the experimented ESP8266 NodeMCU system

Our experiment environment is set as $1 \mathrm{mx} 1 \mathrm{~m}$ to maximize signals interferences also system errors. We install anchor modules in a triangular form to create our network positioning cell. Tag module is installed at a position where it can be held by a human and master anchor is located next to anchor 1 . 


\subsection{Positioning}

The task manager determines tag coordinates to plot respectively their locations graphically. However, the effect of Non-line-ofSight (NLOS) with the different multipath signals, the inconstant antenna gain and the flow of location information add errors to the process. Kalman Filter (KF) reduces the location row flickers and compensate ranging error of our system.

For the purpose of an effective linear positioning [10], KF combines both data of rattling location and operate of estimation status. $\mathrm{KF}$ recursive data processing algorithm consists of two phases, prediction and update phase. Regardless of how efficient KF is, it is no longer a method to overcome nonlinear system restrictions since tags move in random directions. Extended Kalman filter (EKF) is then the solution to adopt in our case of nonlinear tracking system to solve KF positioning limitations [11], [12]. Consequently, to provide a realistic tracking for real-time positioning system EKF is adopted.

\section{ESP8266 NODEMCU SYSTEM VALIDATION}

Primarily, using one tag we test our two-phase algorithm consisting of identification, registration stage and three ranging stages. At each distance ranging phase, we establish communication with no loss at each ESP8266 NodeMCU network point with an error value less than the predefined. We compare then determined positions with the two-phase algorithm to the one phase algorithm calculated positions (reading and ranging only) using a list of three tags installed in the master anchor. Secondarily, we apply the same process for multiple tags.

\subsection{System distance ranging performances}

Firstly, we measure from 0.3 meter to 1 meter anchor/tag distance (we take 8 values of distance and for each distance we take the mean of RSSI for around 100 RSSI values). Secondly, we use fit curve function at each distance ranging to estimate anchor/tag distance. The mean of the three estimated distance values is used for calculation in two-phase algorithm.

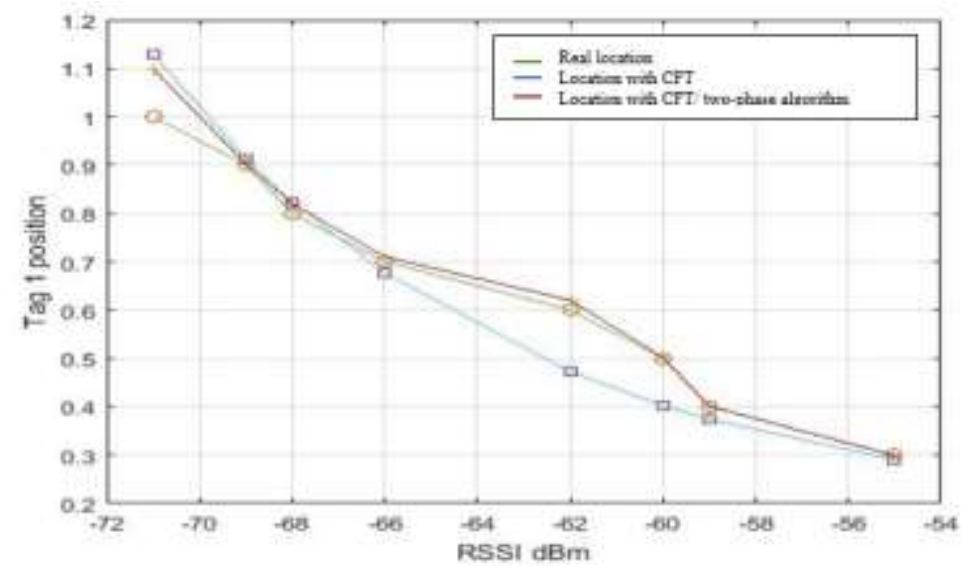

Figure 8: tag distance with CFT (one phase) and CFT/two-phase algorithm

Figure above illustrates distance ranging of a tag using CFT to minimize ranging error [13] for both cases. Positioning with twophase algorithm presents more positioning data, consequently, accurate calculation at each ranging phase compared to one phase ranging algorithm. It also proposes an optimal use of hardware resources at every communication time (between anchor/tag).

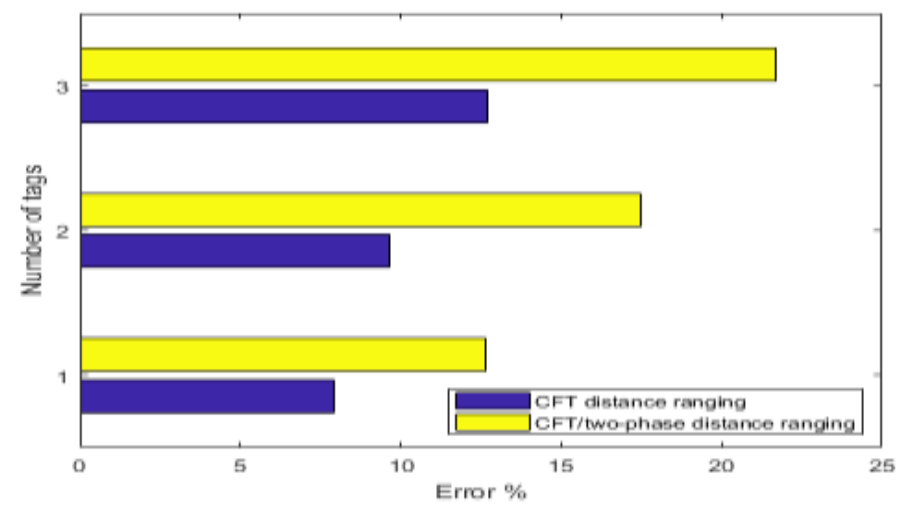

Figure 9: tag ranging error using CFT (one-phase) and CFT/ two-phase algorithm 
Accuracy increases when using the two-phase algorithm as shown in Figure 9. The error of the ESP8266 NODEMCU positioning system with the two-phase algorithm is less than $13 \%$ which is lower compared to the one-phase.

\subsection{Tag identification at the first stage}

We compare the required time for the ESP8266 NODEMCU system with one-phase algorithm and two-phase with a fixed and different identification and registration stage to distance ranging stage ratio during a loop. As the number of activated tags increases, the total required time increases. Moreover, the time parameter is no longer an advantage in case of a high number of activated tags ( $3 \mathrm{rd}$ tag in our case). Generally, in a realistic positioning system, the identification and registration stage are less than the distance ranging stage so that our implemented algorithm still has no time loss. Then this algorithm performs better than the conventional positioning system. We study then the required time for positioning differently using a fixed percentage of activated tags during a loop (Figure 10).

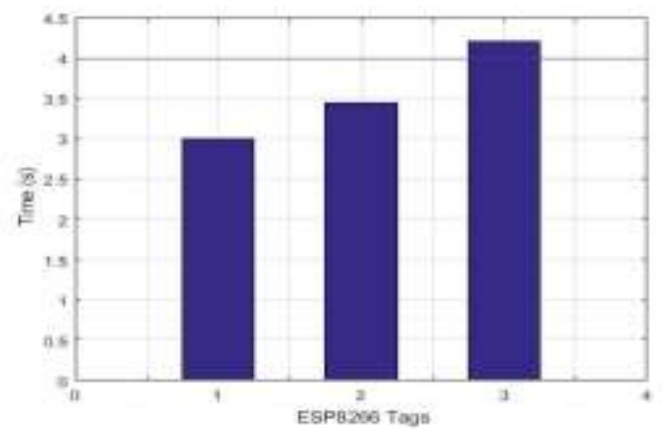

(a)

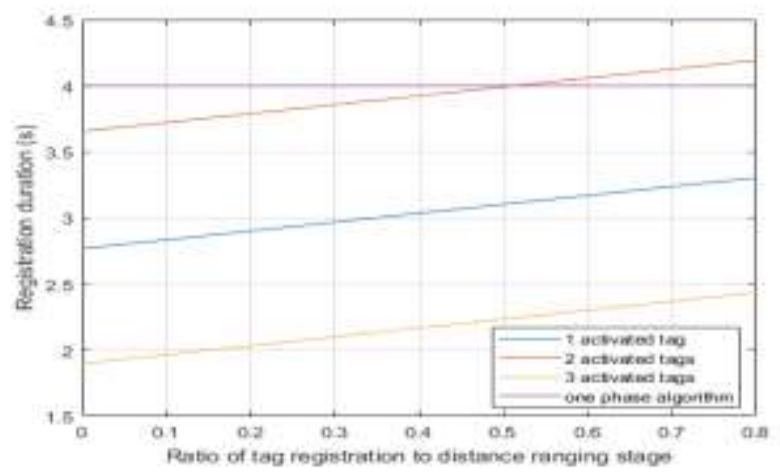

(b)

Figure 10: (a) Time required for positioning with a fixed identification to distance ranging ratio during a loop, (b) required time with a fixed activated tag number during a loop

ESP8266 NODEMCU system with the two-phase algorithm shows a better time management for positioning a defined multi-tag while the mono-phase system algorithm has a constant time regardless of the first stage and distance ranging stage ratio.

\section{CONCLUSION}

In this paper, we propose a tracking system using ESP8266 NODEMCU modules for multi-tag positioning. The proposed system works under a two-phase algorithm; it minimizes the time for the inactive tag at the reading stage to increase the ranging accuracy. By removing the unnecessary signal transmit after the identification and registration phase we lower power consumption and use resources optimally. In addition, the number of registered tags of the tracking affects system efficiency loss. Finally, we consider developing a flexible algorithm to increase the number of identified tags and reduce the huge time consumption in the first stage of the method.

\section{REFERENCES}

1. A. Rai, K. K. Chintalapudi, V. N. Padmanabhan, and R. Sen, "Zee: Zeroeffort crowdsourcing for indoor localization," in Proc. ACM MobiCom, 2012, pp. 293-304.

2. P. Bahl and V. N. Padmanabhan, "RADAR: An in-building RF-based user location and tracking system," in Proc. IEEE INFOCOM, 2000, pp. 775-784.

3. S. Saad and Z. Nakad, "A standalone RFID indoor positioning system using passive tags," IEEE Trans. Ind. Electron., vol. 58, no. 5, pp. 1961-1970, May 2011.

4. P. Yang and W. Wu, "Efficient particle filter localization algorithm in dense passive RFID tag environment," IEEE Trans. Ind. Electron., vol. 61, no. 10, pp. 5641-5651, Oct. 2014.

5. Suguna P. Subramanian, Juergen Sommer, Frank-Peter Zeh, Stephen Schmitt and Wolfgang Rosential, "PBIL-PDR for scalable Bluetooth Indoor Localization", 2009 Third International Conference on Next Generation Mobile Applications, Services and Technologies.

6. J. Chung, M. Donahoe, C. Schmandt, I.-J. Kim, P. Razavai, and M. Wiseman, "Indoor location sensing using geo magnetism," in Proc. ACM MobiSys, 2011, pp. 141-154. 
7. S. Chan and G. Sohn, "Indoor localization using Wi-Fi based fingerprinting and trilateration techniques for LBS applications," International Archives of the Photogrammetry, Remote Sensing and Spatial Information Sciences, vol. 38, p. 4, 2012.

8. M. Brunato and R. Battiti, "Statistical learning theory for location fingerprinting in wireless LANs", Computer Networks, vol. 47, pp. 825-845, 2005.

9. S. Barai, "Estimate distance measurement using NodeMCU ESP8266 NODEMCU based on RSSI technique", proceedings of 2017 IEEE, Cama, Japan, 2018

10. G. F. Welch and G. Bishop, “An Introduction to the Kalman Filter," University of North Carolina, Chapel Hill, NC, USA, Tech. Rep., 1995.

11. Werner, J.; Hakkarainen, A.; Valkama, M., "Estimating the primary user location and transmit power in cognitive radio systems using extended Kalman filters," in Wireless On-demand Network Systems and Services (WONS), 2013 10th Annual Conference on-demand Network Systems and Services (WONS), vol., no., pp.68-73, 18-20 March 2013.

12. Pathirana, P.N.; Savkin, A.V.; Sanjay Jha, "Robust extended Kalman filter applied to location tracking and trajectory prediction for PCS networks," Control Applications, 2004. Proceedings of the 2004 IEEE International Conference on Control Applications, vol.1, no., pp.63,68 Vol.1, 2-4 Sept. 2004

13. Mitigating propagation errors for indoor positioning in wireless sensor networks,"MASS07, Pisa, Italy, 2007. 